\title{
Inégalités géographiques de recours aux soins de santé maternelle au Mali : rôles respectifs des caractéristiques individuelles, familiales et communautaires
}

\author{
Siaka CISSE et Claudine SAUVAIN-DUGERDIL
}

\author{
Institut de Démographie et de Socio-économie (IDESO), \\ Université de Genève. \\ E-mail : siakacisse79@gmail.com
}

\section{Resume}

Contexte: Au Mali, à l'instar de la plupart des pays d'Afrique subsaharienne, les disparités géographiques en matière d'utilisation des services de santé maternelle persistent malgré les politiques nationales visant à améliorer la santé maternelle. Ce travail examine les inégalités communales de recours aux soins de santé maternelle au Mali.

Source de données et Méthodes: Les données de l'Enquête par Grappe à Indicateurs Multiples (MICS, 2009-2010) ont été utilisées et complétées par celles du Recensement Général de la Population et de l'Habitat (RGPH) de 2009. La méthodologie multiniveau a été utilisée à l'aune de l'approche par les capabilités (AC).

Results: Les différences entre communes résultent des inégalités de ressources du contexte (offre de services de santé et degré d'urbanisation), des caractéristiques individuelles et du ménage (éducation, niveau de vie, exposition aux médias, instruction du chef de ménage). Ces différents déterminants contribuent respectivement à la quasi-totalité $(98 \%)$ des inégalités communales de recours à l'accouchement assisté et aux trois quarts de celles en matière de suivi prénatal.

Conclusion: II resort que ce n'est pas seulement les différences individuelles qui modulent l'accès aux soins, mais aussi les caractéristiques de l'entourage familial et social, à savoir, selon la terminologie des capabilités, les facteurs individuels mais aussi collectifs de conversion. L'efficacité des politiques publiques étant évaluée par leur capacité à ouvrir les mêmes capabilités de santé pour tous (Ruger, 2009 ; Bonvin et Rosenstein, 20I I), la persistence des inégalités met en évidence les résultats mitigés des politiques et programmes en matière de santé maternelle au Mali.

Mots Cles: Inégalités de soins de santé, analyse multiniveau, facteurs de conversion, capabilités.

\section{Problématique et hypothèses}

Les études sur le recours aux soins ont essentiellement été abordées "à travers le prisme d'une modélisation théorique du comportement basé sur l'idée que la décision de recourir aux soins pour un individu malade relève d'un choix rationnel. Elles ont le plus souvent traité les questions liées à la demande de soins dans une perspective individuelle en mettant très peu en avant les aspects contextuels et leur influence sur les comportements des individus. Les contextes locaux (par exemples régions, communes, quartiers à l'intérieur d'un pays), et même nationaux, sont souvent considérés comme des ensembles homogènes influençant très peu la demande individuelle de soins. Or les comportements de recours aux soins au niveau individuel sont aussi déterminés par les contextes socioéconomiques et culturels (Franckel et al, 2008). C'est fort de ce constat qu'on voit apparaitre un intérêt grandissant pour la prise en compte de la dimension communautaire, notamment ces vingt dernières années. Ainsi, certains chercheurs de domaines divers et singulièrement en épidémiologie sociale cherchent à intégrer les effets du contexte de résidence (niveau socioéconomique, caractéristiques de la communauté, offres de services, etc.) pour chercher à comprendre les comportements des individus en matière de santé (Chaix et Chauvin, 2002 ; Stephenson et al. 2006 ; Grady et al., 1993 ; Chacko, 2001 ; DeGraff et al., 1997 ; Entwisle et al., 1989 ; Entwisle et Mason, 1985 ; Manda, 1998 ; Congdon, 1995 ; Pebley et al., 1996 ; Vallée, 2009, etc.).

Entwisle et Mason (1985), cherchant à décrire les déterminants micro et macro de la demande d'enfant dans quinze pays concernés par l'enquête mondiale sur la fécondité, arrivaient à la conclusion que le produit national brut par habitant et l'effort entrepris dans les programmes de planification familiale influençaient les différences des niveaux moyens de fécondité entre les pays. En milieu rural égyptien, Entwisle et al. (1989) s'intéressaient à l'effet des 
caractéristiques du village (la structure de l'économie, la modernisation de l'agriculture, le niveau de participation scolaire et l'offre de services de planification familiale). Leurs résultats ont indiqué que le comportement contraceptif variait systématiquement avec les caractéristiques du village. De même, l'étude de Grady et al. (1993) relative aux décisions de recourir à la contraception, parmi les femmes blanches mariées au États-Unis, a mis en évidence l'importance des caractéristiques de la communauté dans les choix contraceptifs des femmes. Les localités ayant un meilleur statut socioéconomique ainsi qu'un accès facile à l'information et aux services de planification familiale sont celles où les femmes sont plus susceptibles d'utiliser les méthodes contraceptives de manière efficace. Un résultat similaire a été observé aux Philippines sur l'utilisation différentielle de la contraception selon le niveau de développement économique et de dotation en infrastructures de la communauté de résidence (DeGraff et al., 1997).

Pour le cas spécifique des soins obstétricaux, Magadi et al. (2007), dans leur travail sur 21 pays de l'Afrique Subsaharienne, montrent que les politiques nationales (politiques de partage des coûts, disponibilité et accessibilité des services de santé maternelle) expliquent 7 à $15 \%$ des variabilités totales des soins prénatals et de la prise en charge de l'accouchement entre les pays. Pareillement, Stephenson et al. (2006) ont identifié les influences des caractéristiques de la communauté sur la décision d'accoucher dans un établissement de santé dans six pays africains (Côté d'lvoire, Burkina Faso, Ghana, Kenya, Malawi et Tanzania). Ainsi, le pourcentage de femmes ayant une scolarisation secondaire apparait significativement associé à l'accouchement assisté au Malawi, au Kenya et au Ghana. À l'inverse, le nombre moyen d'enfants par femme avait une influence négative sur le recours à l'accouchement dans un centre de santé, quel que soit le pays. Selon les auteurs, les collectivités ayant une fécondité plus élevée ont le plus souvent un faible niveau de développement économique, mais pourraient aussi avoir des attitudes plus conservatrices en matière d'utilisation des services. Une fécondité élevée peut exprimer aussi un manque de services de santé génésique. Dans le cas du Nigeria, Babalola et Fatusi (2009) montrent que, au-delà de l'éducation de la femme et du niveau socio-économique du ménage, les caractéristiques de la communauté, en matière d'urbanisation et de densité des médias, sont des prédicteurs importants de l'utilisation des services. Un résultat similaire a été observé par Ononokpono, (20I5) dans le même contexte : la propension individuelle à accoucher dans les centres de santé varie fortement selon les proportions moyennes de femmes éduquées et du niveau de pauvreté de la zone de résidence.

Ainsi, au-delà des différences d'offre de services, le contexte dans lequel vivent les individus façonne d'une certaine manière leurs attitudes et comportements. C'est ce qui peut expliquer, en partie, le fait que des individus de différentes zones géographiques ont des propensions différentes à recourir aux soins prénatals et obstétricaux. Ainsi, les femmes d'une commune ou d'un quartier spécifique peuvent être plus semblables dans leurs comportements. De même, les personnes ayant des caractéristiques personnelles similaires, par exemple un niveau élevé d'éducation, peuvent avoir différents états de santé en raison des différences culturelles, économiques, politiques, climatiques, historiques ou environnementales inhérentes au contexte local (Merlo et al, 2005). Par conséquent, il convient de mieux distinguer les inégalités de recours aux soins dues à l'offre de service (ressources du contexte), et à la composition socioéconomique de la population féminine (facteurs collectifs de conversion), de celles liées aux caractéristiques individuelles et familiales (facteurs individuels ou familiaux de conversion). II s'agit alors de chercher à mieux comprendre les interactions qui s'opèrent entre les facteurs de conversion collectifs et individuels ou familiaux qui constituent des moyens d'expansion des capacités réelles à utiliser l'offre de services en tant que ressources du contexte.

Au Mali, à l'instar de la plupart des pays d'Afrique au Sud du Sahara, les disparités géographiques en matière d'utilisation des services de santé maternelle persistent. Les régions du Nord enregistrent la proportion de femmes ayant effectué un suivi prénatal la plus faible (respectivement $32 \%, 43 \%$ et $58 \%$ à Kidal, Tombouctou et Gao contre $64 \%$; $66 \% ; 71 \% ; 70,5 \% 75 \%$ et $94 \%$ respectivement à Kayes, Mopti, Koulikoro, Sikasso et Bamako). Les femmes de Kidal (20\%), Tombouctou (26\%) et de Gao (34\%) et Mopti (34\%) ont le plus fréquemment accouché sans l'assistance de personnel formé comparées aux femmes des régions du Sud (EDSMIV, 2006). Le maintien de ces inégalités géographiques malgré les politiques nationales visant à améliorer la santé maternelle soulève des interrogations. Quels sont les déterminants associés aux inégalités géographiques de recours aux soins prénatals et obstétricaux au Mali ? Est-ce le résultat d'inégalités de dotations ou de ressources du contexte (l'offre de services) ou de la capacité et de la volonté des individus à recourir aux soins (facteurs individuels/familiaux et collectifs de conversion) ? Comment les facteurs individuels ou familiaux interagissent avec les ressources du contexte dans la demande de soins? 


\section{Hypothèses de recherche}

Nous analysons ici les inégalités communales de recours aux soins prénatals et obstétricaux à l'aune de l'approche par les capabilités, en postulant qu'elles résultent d'une inégale répartition de capabilités de santé maternelle, c'est-à-dire des différences de capacité pour les mères d'être en bonne santé pour paraphraser Ruger (2009). En d'autres termes les femmes vivant dans différentes communes n'ont pas les mêmes libertés réelles de recourir aux soins quand elles le désirent eu égard aux différences de caractéristiques individuelles et familiales (facteurs individuels ou familiaux de conversion), des spécificités locales en matière de composition socioéconomique de la population féminine (facteurs collectifs de conversion) et de l'offre de services sanitaires (dotations ou ressources du contexte).

Les caractéristiques individuelles et familiales, examinées ici à travers le niveau d'éducation, le degré d'exposition aux médias, le niveau de vie du ménage et l'éducation du chef de ménage constituent des facteurs individuels ou familiaux de conversion qui influencent l'accès aux ressources du contexte. Ces facteurs individuels ou familiaux sont cependant modulés par les différences géographiques de composition de la population féminine, considérées comme des facteurs collectifs de conversion.

$\mathrm{HI}$ : Les inégalités entre les femmes de différentes communes en matière de recours aux soins résulteraient des différences de leur capacité individuelles et familiales à convertir l'offre de services de santé en un recours effectif aux soins. En effet, les femmes d'un niveau d'éducation élevé, plus exposées aux média et vivant dans les ménages avec un niveau de vie élevé sont plus susceptibles de recourir effectivement aux soins.

$\mathrm{H} 2$ : Les femmes vivant dans les communes disposant de meilleures dotations ou ressources du contexte (plus équipées en infrastructures sanitaires) sont plus avantagées en termes de recours aux soins. Mais le recours effectif aux soins est influencé par la composition socioéconomique de la population féminine (niveaux communaux d'éducation de la femme et des conditions de vie du ménage). Ainsi, les communes ayant une proportion élevée de femmes éduquées ou un pourcentage élevé de ménages situés parmi les plus riches du pays sont celles où les femmes ont une plus grande propension à recourir aux soins prénatals et obstétricaux.

H3 : L'influence des facteurs individuels ou familiaux de conversion sur la propension à utiliser les services de santé maternelle varie d'un contexte de résidence à un autre en raison des différences communales de composition socioéconomique de la population féminine et de dotations ou ressources.
En effet, l'influence de l'éducation et du niveau d'instruction des femmes sur la propension à recourir aux soins de santé pendant la grossesse et à l'accouchement varie selon le milieu de résidence.

\section{Données}

Les données utilisées, dans cette étude, proviennent de l'Enquête par Grappe à Indicateurs Multiples (MICS) réalisée au Mali en 2009-2010 par la Cellule de Planification et de Statistique du Ministère de la Santé, du développement social et la promotion de la famille et l'Institut National de la Statistique, avec l'appui technique et financier de l'UNICEF, de la Coopération Suédoise, de l'Union Européenne, de la Banque Mondiale et de l'Agence des États Unis d'Amérique pour le Développement International (USAID). Inscrite dans un programme international d'enquêtes auprès des ménages initié par l'UNICEF, cette enquête fournit des informations sur la situation des enfants et des femmes permettant de suivre les progrès dans la réalisation des Objectifs du Millénaire pour le Développement (OMD) et d'autres engagements internationaux. Nous complétons ces données par celles du Recensement Général de la Population et de l'Habitat du Mali réalisé en 2009 par le Bureau Central du Recensement (BCR) du Mali.

\section{Population cible et variables d'intérêt}

La population d'étude est constituée des femmes âgées de 15-49 ans en union et ayant eu leur dernière naissance au cours des deux dernières années précédant l'enquête.

Comme variables d'intérêts, nous retenons deux indicateurs clés de la santé maternelle que sont la consultation prénatale et l'assistance à l'accouchement en raison de leur importance dans le maintien de la santé de la mère pendant sa période de procréation. Le questionnaire adressé aux femmes de 15-49 ans contenait un module "Santé de la mère et du nouveau-né". Dans ce module, la question suivante : "Avez-vous reçu des soins prénatals au cours de la grossesse de (nom) ?" était posée aux femmes qui ont eu une naissance vivante dans les deux années ayant précédé la date de l'interview. C'est cette question qui permet d'appréhender la consultation prénatale avec comme modalité Oui ou Non. Ensuite, il a été demandé aux mêmes femmes le lieu où elles avaient accouché de leur dernier enfant : "Ou avez-vous accouché de (nom) ?". Cette dernière variable a été recodée de manière à avoir deux modalités (Oui ou Non). Les femmes qui ont accouché dans les centres de santé publique ou privé sont considérées comme ayant été assistées par un personnel de santé qualifié. Ce sont dans ces centres de santé qu'on retrouve les médecins, infirmières, sages-femmes et matrones, lesquels sont supposés 
être formés et qualifiés, même si certains d'entre eux n'ont pas toute la qualification requise. Ces deux variables constituent donc les variables dépendantes avec respectivement $0,7 \% \quad(76$ sur $\quad 10157$ observations) et $0,8 \%$ (85 sur I $0 \mid 57$ observations) de valeurs manquantes.
Un peu plus du quart $(26,1 \%)$ des femmes âgées de 15-49 ans en union n'ont pas consulté pendant leur dernière grossesse intervenue au cours des deux années précédant l'enquête. En ce qui concerne l'assistance à l'accouchement, près d'une femme sur deux a accouché sans l'assistance d'un personnel de santé $(46,3 \%)$ en 2010.

Tableau I: Distribution des mères de I 5-49 ans selon leur recours à la consultation prénatale et à l'assistance à l'accouchement

\begin{tabular}{|c|c|c|c|c|c|c|}
\hline \multirow[b]{2}{*}{ Modalités } & \multicolumn{3}{|c|}{ Consultation prénatale } & \multicolumn{3}{|c|}{ Accouchement assisté } \\
\hline & Effectifs & $\%$ & $\%$ valide & Effectifs & $\%$ & $\%$ valide \\
\hline Non & 2630 & 25,9 & 26,1 & 4664 & 45,9 & 46,3 \\
\hline Oui & 7451 & 73,4 & 73,9 & 5408 & 53,2 & 53,7 \\
\hline NA & 76 & 0,7 & & 85 & 0,8 & \\
\hline Total & 10157 & 100,0 & 100,0 & 10157 & 100,0 & 100,0 \\
\hline
\end{tabular}

\section{Variables explicatives}

Les variables explicatives retenues au niveau individuel et du ménage sont le niveau d'instruction de la femme, son exposition aux médias, le niveau de vie du ménage et l'éducation de son chef. Déjà disponible dans la base de données, le niveau de vie est un indicateur classique de proxy du niveau de richesse des ménages. Elle est construite à partir de questions relatives aux matériaux des murs, du sol et du toit des logements, à la possession de biens durables de consommation et à l'accès à l'eau et aux installations sanitaires. En sus, d'autres variables sont utilisées pour servir de contrôle à celles précédemment citées : il s'agit au niveau individuel, de l'âge de la femme et de son groupe ethnique, au niveau ménage du sexe du chef de ménage. La construction, le recodage et la description de l'ensemble de ces variables sont présentés dans le tableau ci-après.

Tableau 2 : Tableau descriptif des variables du niveau individuel et du ménage

\begin{tabular}{|c|c|c|c|c|c|}
\hline Variables & Catégories initiales & Recodage & Des & tion & \\
\hline \multirow[b]{2}{*}{$\begin{array}{l}\text { Age de la } \\
\text { femme }\end{array}$} & \multirow[b]{2}{*}{$>=15$ ans $\&<=49$ ans } & \multirow[b]{2}{*}{$\begin{array}{l}\text { I } 5-19 \rightarrow \text { Ado/jeune } \\
20-34 \rightarrow \text { Moins jeunes } \\
35 \text { ou plus } \rightarrow \text { Adultes }\end{array}$} & Effectifs & $\%$ & $\begin{array}{l}\% \\
\text { valide }\end{array}$ \\
\hline & & & $\begin{array}{l}1150 \\
7128 \\
1879\end{array}$ & & $\begin{array}{l}11,3 \\
70,2 \\
18,5\end{array}$ \\
\hline \multirow{5}{*}{$\begin{array}{l}\text { Fréquence } \\
\text { d'écoute de la } \\
\text { radio \& du } \\
\text { temps passé } \\
\text { devant la télé }\end{array}$} & I. Pratiquement chaque jour & I (Radio) ou I (Télé) $\rightarrow$ I & 1579 & 15,5 & 15,6 \\
\hline & $\begin{array}{l}\text { 2. Au moins une fois par } \\
\text { semaine }\end{array}$ & 2 (Radio) ou 2 (Télé) $\rightarrow 2$ & 1379 & 13,6 & 13,6 \\
\hline & 3. Moins d'une fois par semaine & 3 (Radio) ou 3 (Télé) $\rightarrow 3$ & $|22|$ & 12,0 & 12,0 \\
\hline & 4. Pas du tout & 4 (Radio) ou 4 (Télé) $\rightarrow 4$ & 5962 & 58,7 & 58,8 \\
\hline & Manquant & $\mathrm{nt}->\mathrm{NA}$ & 16 & 0,2 & \\
\hline \multirow{3}{*}{$\begin{array}{l}\text { Niveau } \\
\text { d'instruction } \\
\text { de la femme }\end{array}$} & I. Aucun & & 8131 & & 80,1 \\
\hline & 2. Primair & & $130 \mid$ & & 12,8 \\
\hline & 3. Secondaire ou plus & & 725 & & 7,1 \\
\hline \multirow{7}{*}{ Ethnie } & \multirow{7}{*}{$\begin{array}{l}\text { Bambara ; Malinke ; } \\
\text { Peulh/foulfoulbé; Sonraî/djerma; } \\
\text { Sarakole/soninke/marka; } \\
\text { Kassonké; Sénoufo; Dogon; } \\
\text { Maure; Tamacheq; Bobo; } \\
\text { Dafing; Minianka; Haoussa; } \\
\text { Samogo; Bozo; Arabe; Autres } \\
\text { ethnie africaines; Autres ethnies }\end{array}$} & I. Ban & 2793 & 27,5 & 27,5 \\
\hline & & 2. Malinke/Kassonké & 83 & 8,2 & 8,2 \\
\hline & & 3. Peulh/foulfoulbé & 1405 & 13,8 & 13,8 \\
\hline & & 4. Sonrai/djerma/hac & 1111 & 10,9 & 10,9 \\
\hline & & 5. Sarakole/soninke/marka & 1109 & 10,9 & 10,9 \\
\hline & & ufo/minianka & 1099 & 10,8 & 10,8 \\
\hline & & 7. Dogon & 549 & 5,4 & 5,4 \\
\hline
\end{tabular}




\begin{tabular}{|c|c|c|c|c|c|}
\hline \multirow[t]{4}{*}{ Variables } & Catégories initiales & \multirow{4}{*}{$\begin{array}{l}\text { Recodage } \\
\text { 8. Maure/tamacheq/Arabe } \\
\text { 9. Autres } \\
\text { NA }\end{array}$} & \multicolumn{3}{|c|}{ Description } \\
\hline & étrangères; Autres ethnies du & & 616 & 6,1 & 6,1 \\
\hline & Mali ; Manquant. & & 630 & 6,2 & 6,2 \\
\hline & & & 10 & 0,1 & \\
\hline \multirow{3}{*}{$\begin{array}{l}\text { Niveau de vie } \\
\text { du ménage }\end{array}$} & \multirow{3}{*}{$\begin{array}{l}\text { I.Très pauvre, } 2 \text {. Pauvre, } 3 \text {. } \\
\text { Moyen, } 4 \text {. Riche, } 5 \text {. Très riche }\end{array}$} & I ou $2 \rightarrow$ Pauvre & 4362 & & 42,9 \\
\hline & & 3 ou $4 \rightarrow$ Moyen & 4236 & & $4 \mathrm{I}, 7$ \\
\hline & & $5 \rightarrow$ Riche & 1559 & & 15,3 \\
\hline \multirow{4}{*}{$\begin{array}{l}\text { Niveau } \\
\text { d'instruction } \\
\text { du CM }\end{array}$} & I. Aucun & I. Aucun & 8163 & 80,4 & 80,7 \\
\hline & 2. Primaire & 2. Primaire & 1026 & 10,1 & 10,1 \\
\hline & 3. Secondaire ou plus & 3. Secondaire ou plus & 920 & 9,1 & 9,1 \\
\hline & Manquant & Manquant $->N A$ & 48 & 0,5 & \\
\hline \multirow{2}{*}{ Sexe du CM } & Homme & & 9713 & & 95,6 \\
\hline & Femme & & 444 & & 4,4 \\
\hline Total & & & 10157 & & 100,0 \\
\hline
\end{tabular}

Les variables de dotations du contexte sont constituées du milieu de résidence, de la région et du ratio de centres de santé pour dix mille habitants. Les niveaux moyens d'éducation de la femme et de conditions de vie du ménage dans la commune sont utilisés pour appréhender la composition socioéconomique de la population féminine (tableau 3 ). Le milieu et la région de résidence sont utilisés tels que définis dans la base de données. Par contre, nous avons calculé le ratio des centres de santé avec les données du recensement en rapportant le nombre de centres de santé de chaque commune à sa population. L'indicateur obtenu, regroupé en trois catégories d'effectifs similaires, a été ensuite inséré dans notre base de données d'analyse. Les niveaux moyens d'éducation et de conditions de vie des ménages dans la commune sont également répartis en trois catégories. En raison du fort aplatissement de la distribution de ces variables vers les petites valeurs, les premières catégories comprennent respectivement les communes dans les lesquelles aucune femme n'a atteint le niveau secondaire et ne vit dans un ménage situé parmi les $20 \%$ les plus riches du pays.

Tableau 3 : Récapitulatif des variables contextuelles et de composition communale de la population féminine

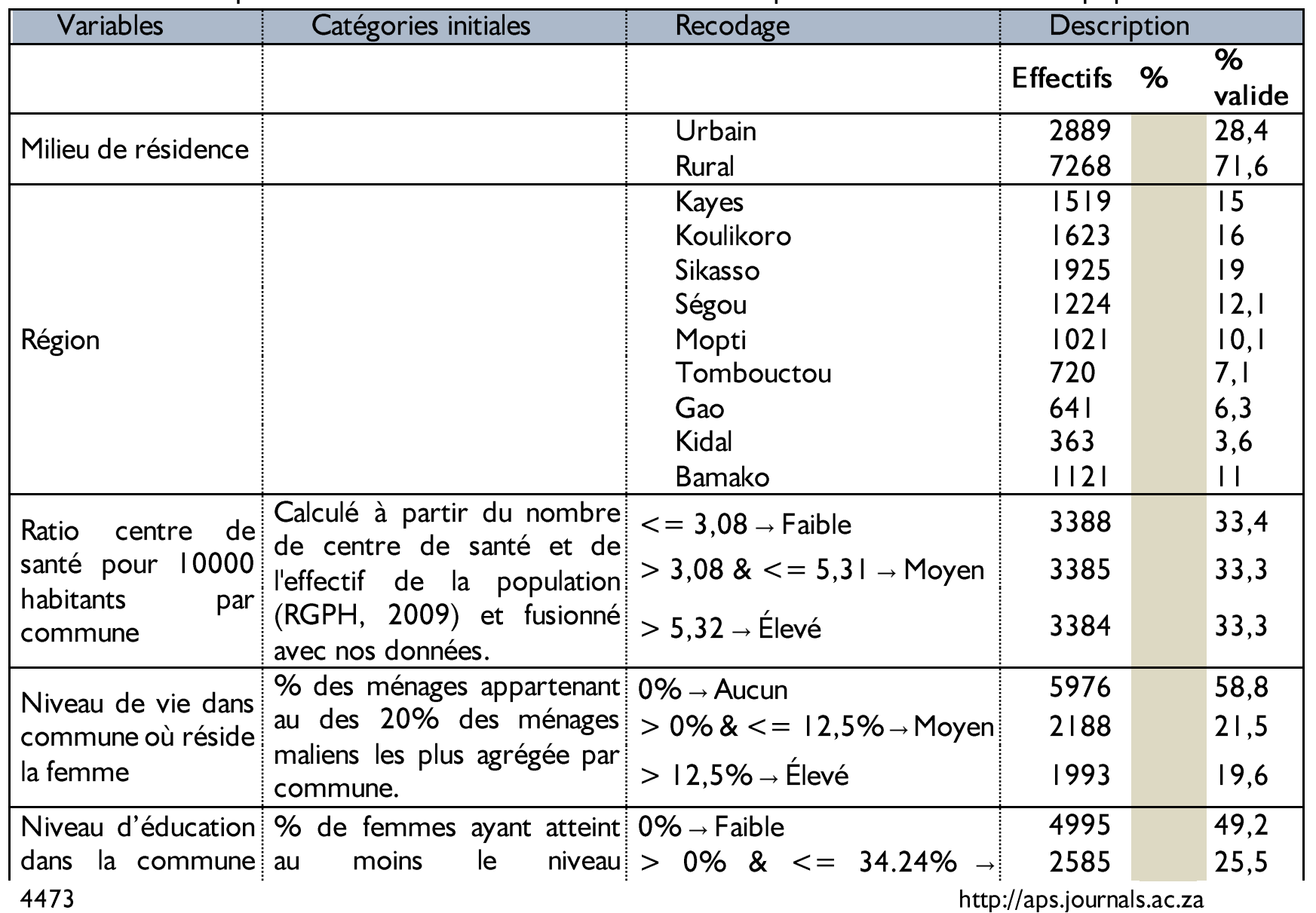




\begin{tabular}{|c|c|c|c|c|}
\hline Variables & Catégories initiales & Recodage & \multicolumn{2}{|c|}{ Description } \\
\hline où réside la femme & $\begin{array}{l}\text { secondaire agrégée par } \\
\text { commune }\end{array}$ & $\begin{array}{l}\text { Aucun } \\
>34.24 \% \rightarrow \text { Élevé }\end{array}$ & 2577 & 25,4 \\
\hline Total & & & 10157 & 100 \\
\hline
\end{tabular}

\section{Méthode d'analyse}

Les méthodes d'analyse utilisées sont à la fois descriptives et explicatives. D'un point de vue descriptif, deux méthodes sont appliquées. La première porte sur l'analyse bivariée basée sur le croisement des variables à expliquer (recours à la consultation prénatale et à l'accouchement assisté) avec chacune des variables explicatives (test de Khideux). Cette analyse est stratifiée par milieu de résidence. Le niveau de significativité dans le cadre de nos analyses est fixé à $5 \%$.

Pour ce qui est de l'analyse explicative, nous utilisons la régression multiniveau pour déterminer les variabilités intercommunales (420 communes) de recours aux soins liées aux caractéristiques individuelles et familiales (facteurs individuels ou familiaux de conversion), aux différences communales de composition socioéconomique de la population féminine (facteurs collectifs de conversion) et aux ressources du contexte (offre de service de santé) à l'aide du package Ime4 du logiciel R. Apparue au milieu des années 1980, cette méthode permet l'estimation simultanée des résidus au niveau des individus et de la variance des effets aléatoires au niveau des groupes (Vallée, 2009).

Étant donné la nature de la variable dépendante (qualitative et dichotomique), nous recourons au modèle de régression logit multiniveau dont l'équation simplifiée est donnée par :

$\operatorname{logit}\left(\pi_{i j}\right)=\ln \left(\frac{P\left(Y_{i j}=1 \mid x_{i j}, u_{j}\right)}{P\left(Y_{i j}=0 \mid x_{i j}, u_{j}\right)}\right)=\alpha_{1}+$ $\sum_{k=0}^{k} \beta_{k} x_{k i j}+u_{j}$

$u_{j} \sim N\left(0, \sigma^{2}\right) j=1,2, \ldots, j i=1,2, \ldots, n j$

Où $\pi_{i j}$ est la probabilité conditionnelle que la femme i dans la commune $j$ recoure aux soins de santé maternelle (prénatals ou accouchement assisté) ;

$Y_{i j}$ la valeur de la variable dichotomique (dans notre cas, I si recours aux soins de santé maternelle et 0 si non) de la i ${ }^{\text {ème }}$ femme dans la je ${ }^{\text {ème }}$ commune ;

$x_{k i j}$ représente les variables explicatives utilisées dans le modèle ;

$u_{j}$ est l'effet aléatoire de la jème ${ }^{\text {commune et est }}$ supposé suivre une distribution normale avec une moyenne nulle et la variance égale à $V\left(u_{j}\right)=\sigma^{2}$ $k$ est le nombre des variables explicatives dans le modèle, $\alpha_{1}$ l'ordonnée à l'origine (intercept) et $\beta_{k}$ représente le coefficient de la régression pour la $k^{\text {ème }}$ variable explicative. Dans le cas du modèle nul, $k$ prend la valeur zéro et on aura donc $\operatorname{logit}\left(\pi_{i j}\right)=\alpha_{1}+u_{j}$;

Dans le modèle logit, la variance de niveau individuel est égale à $\frac{\pi^{2}}{3}$ (c'est-à-dire à 3,29). La part de la variabilité du recours aux soins de santé chez les femmes enceintes et parturientes due aux différences de communes vaut $V P C=\operatorname{ICC}=\left(\frac{\sigma^{2}}{\sigma^{2}+\frac{\pi^{2}}{g}}\right) * 100$. Dans ce cas, si $\sigma^{2}$ est nulle, alors il n'existe aucune disparité inter-commune. En revanche, si $\sigma^{2}$ est significativement supérieure à 0 , on peut conclure à une différence entre les unités géographiques. Cette part de variabilité due aux différences de commune $(V P C)$ n'est pas à confondre avec la variation en proportion de la variance intercommunautaire liée au passage à un modèle supplémentaire (PCV).

Ce type d'analyse permet de mesurer l'influence des facteurs communautaires et individuels sur les inégalités géographiques de recours aux soins prénatals et obstétricaux. La prise en compte du niveau communautaire dans l'analyse offre l'occasion de mettre en évidence les risques de non recours des soins associés à la structure sociale et à l'écologie (Stephenson et al, 2006). Autrement dit, cette méthode permet d'examiner s'il y a une différence entre les unités géographiques (communes dans notre cas) en matière d'utilisation des soins de santé maternelle et de mettre en évidence les rôles respectifs des profils sociodémographiques des femmes et de leur ménage d'une part (effet de composition) et des caractéristiques contextuelles d'autre part (effet du contexte : par exemples le niveau de développement économique, la présence et la qualité des services de santé, les normes sociales et culturelles). L'intégration dans le même modèle des caractéristiques individuelles avec celles agrégées évite de commettre l'erreur atomiste qui consiste à isoler l'individu sans faire intervenir les contraintes du milieu dans lequel il vit, ainsi que l'erreur écologique qui consiste à inférer à l'individu une association statistique observée à un niveau agrégé (Courgeau, 2004 ; Von Korff et al. ( 1992). " L'articulation, dans un même modèle, de données collectées à des niveaux différents (individu, groupes d'individus, souspopulations, etc.) permet de distinguer correctement 
l'effet des caractéristiques individuelles de l'effet des caractéristiques des divers niveaux d'agrégation considérés, ainsi que d'un effet aléatoire propre à chaque niveau »(Nganawara, $2016: 23$ ).

Cette méthode explicative présente l'intérêt de pouvoir comparer la variance de niveau groupe (commune) avant et après l'introduction des caractéristiques individuelles/familiales et contextuelles (différences de composition de la population féminine et d'offre de services de santé) dans la régression et de quantifier leurs parts respectives dans la variabilité intercommunale de recours aux soins. Elle est, par ailleurs, utile pour déterminer si les variations inter-intercommunale repérées concernent l'ensemble des femmes, ou certaines d'entre elles aux profils particuliers. Ce type d'analyse conduit ainsi à une meilleure identification des populations à risque, utile lors de l'élaboration des programmes de santé (Chauvin, 2002).

\section{Résultats des analyses \\ Analyse descriptive}

L'âge de la femme n'influence pas significativement I'utilisation des services de santé maternelle en milieu urbain, ceci quel que soit l'indicateur de santé maternelle considéré. Par contre, en milieu rural, le recours aux soins prénatals et obstétricaux est globalement plus fréquent parmi les adolescentes (I5-19 ans) que parmi les femmes moins jeunes. Cette association entre l'âge de la femme et l'utilisation des services de santé, en milieu rural, varie selon le type de soins et permet de distinguer deux groupes de femmes. Les adolescentes et les jeunes (moins de 35 ans) utilisent plus les services de santé pendant leur grossesse par rapport aux femmes adultes (35 ans ou plus), alors que pour l'accouchement, ce sont les adolescentes qui se distinguent de toutes les autres en recourant plus à l'assistance d'un personnel de santé.

Le niveau d'instruction de la femme est significativement associé à l'utilisation des services de santé pendant la grossesse et l'accouchement. On observe une hausse des prévalences de consultation prénatale et d'accouchement assisté proportionnellement à une amélioration du niveau d'éducation de la femme, aussi bien en milieu urbain qu'en milieu rural. En d'autres termes, plus le niveau d'instruction atteint est élevé, plus les femmes recourent aux soins. Cependant, quelle que soit la composante de santé maternelle retenue, les écarts de recours aux soins selon le niveau d'instruction sont beaucoup plus marqués en milieu rural qu'ils ne le sont en milieu urbain. La minorité des femmes rurales ayant atteint le niveau secondaire ou plus se distingue particulièrement des autres par un recours aux soins plus fréquent.
L'utilisation des services de santé maternelle est nettement moins élevée parmi les femmes vivant dans les ménages pauvres. La prévalence de consultation prénatale et d'accouchement assisté augmentent suivant le gradient du niveau de vie du ménage, et cela quel que soit le milieu de résidence. Les disparités de recours aux soins selon le niveau de vie sont cependant plus prononcées en milieu urbain qu'elles ne le sont en milieu rural.

Le suivi prénatal et l'accouchement assisté sont plus fréquents parmi les femmes plus exposées aux médias (qui regardent la télévision ou qui écoutent la radio pratiquement chaque jour), quelle que soit la dimension de santé maternelle retenue, avec cependant quelques spécificités selon le milieu. En milieu urbain, les femmes qui n'ont pas du tout accès aux médias ont moins recours aux soins par rapport à toutes les autres. Ce qui n'est pas le cas en milieu rural où le suivi de la grossesse et l'accouchement assisté suivent le gradient de la fréquence d'exposition des femmes aux médias : celles ayant quotidiennement accès aux médias, celles qui y sont moyennement exposées (au moins une fois ou moins d'une fois par semaine) ont un des prévalences plus élevées par rapport à celles qui n'ont pas du tout accès aux médias.

Le niveau de recours aux soins prénatals et à l'accouchement assisté est plus faible chez les femmes appartenant au groupe ethnique Maures/Tamacheqs/Arabes, quel que soit le milieu de résidence. Elles sont suivies d'assez près par celles du groupe Sonrai/Djerma/Haoussa pour l'accouchement assisté. Bien que les femmes dogons soient parmi celles qui recourent le plus aux soins prénatals, dans les deux milieux, et à l'accouchement assisté en milieu urbain, elles se situent parmi celles qui accouchent le moins avec l'assistance d'un personnel de santé en milieu rural. Le même constat est fait, mais dans une moindre mesure, pour les femmes du groupe ethnique Peulh/foulfoulbé.

Comme on pouvait s'y attendre, le District de Bamako se distingue par des prévalences de consultation prénatale et d'assistance à l'accouchement considérablement plus élevées que dans les autres régions du pays. C'est surtout pour l'assistance à l'accouchement que la différence des femmes de la capitale avec les autres est plus marquée. Outre le gap avec le district de Bamako, les autres régions diffèrent aussi en matière d'utilisation des services de santé maternelle. De façon générale, la région de Kidal, suivie de Tombouctou sont celles où les femmes recourent le moins aux soins prénatals et obstétricaux. Les différences régionales de recours aux soins sont encore plus fortes pour l'accouchement assisté, pour lequel deux groupes de régions se distinguent par leur prévalence 
particulièrement faible. Dans la région de Kidal seulement une femme sur dix accouche avec l'assistance d'un personnel de santé. Dans le second groupe composé des régions de Tombouctou, Mopti et Gao, en moyenne, un tiers des femmes accouchent dans les centres de santé. C'est surtout les femmes rurales, vivant dans ces deux groupes de régions, qui présentent beaucoup de désavantages en termes de recours à l'accouchement assisté. Elles sont suivies d'assez près par les parturientes rurales résidant à Ségou et Kayes, deux régions du CentreSud et Sud-Ouest dans lesquels, en revanche les femmes urbaines se situent parmi celles ayant les prévalences de recours à l'accouchement les plus élevées.

Tableau 4 : Prévalence de consultation prénatale et de l'assistance à l'accouchement selon les caractéristiques individuelles, du ménage et contextuelles

\begin{tabular}{|c|c|c|c|c|c|c|c|c|c|c|c|c|}
\hline \multirow{2}{*}{$\begin{array}{l}\text { Caractéristiques } \\
\text { individuelles, du ménage } \\
\text { et contextuelles }\end{array}$} & \multicolumn{6}{|c|}{ Consultation prénatale } & \multicolumn{6}{|c|}{ Accouchement assisté } \\
\hline & \multicolumn{2}{|c|}{ Urbain } & \multicolumn{2}{|c|}{ Rural } & \multicolumn{2}{|c|}{ Total } & \multicolumn{2}{|c|}{ Urbain } & \multicolumn{2}{|c|}{ Rural } & \multicolumn{2}{|c|}{ Total } \\
\hline Groupe d'âge & $\%$ & Eff. & $\%$ & Eff. & $\%$ & Eff. & & Eff. & & Eff. & $\%$ & Eff. \\
\hline Ado. $(15-19)$ & 88,4 & 354 & 69,1 & 784 & 75,1 & 1138 & 83,0 & 353 & 48,9 & 784 & 59,5 & 1137 \\
\hline Jeunes $(20-34)$ & 89,4 & 2040 & 68,4 & 5037 & 74,5 & 7077 & $\mid 81,8$ & 2037 & 42,2 & 5033 & 53,6 & 7070 \\
\hline Adultes (35-49) & 89,2 & 463 & 65,1 & 1403 & 71,1 & 1866 & 81,6 & 462 & 40,3 & 1403 & 50,5 & 1865 \\
\hline Pr. Chi2(2) & \multicolumn{2}{|c|}{ NS } & \multicolumn{2}{|c|}{ *** } & \multicolumn{2}{|c|}{ **** } & \multicolumn{2}{|c|}{ NS } & \multicolumn{2}{|c|}{$* * *$} & \multicolumn{2}{|c|}{$* * *$} \\
\hline \multicolumn{13}{|l|}{ Niveau d'instruction } \\
\hline Aucun & 84,3 & 1757 & 66,0 & 6318 & 70,0 & 8075 & $\mid 74,8$ & 1753 & 40,1 & 6315 & 47,6 & 8068 \\
\hline Primaire & 96,4 & 550 & 78,1 & 736 & 85,9 & 1286 & 90,2 & 549 & 54,6 & 735 & 69,8 & 1284 \\
\hline Secondaire ou plus & 97,8 & 550 & 91,8 & 170 & 96,4 & 720 & 96,4 & 550 & 81,8 & 170 & 92,9 & 720 \\
\hline Pr. Chi2(2) & \multicolumn{2}{|c|}{ **** } & \multicolumn{2}{|c|}{ **** } & \multicolumn{2}{|c|}{ *** } & \multicolumn{2}{|c|}{ **** } & \multicolumn{2}{|c|}{ **** } & \multicolumn{2}{|c|}{$* * *$} \\
\hline Niveau de vie & & & & & & & & & & & & \\
\hline Pauvre & 63,7 & 281 & 60,2 & 4045 & 60,4 & 4326 & |39,I & 281 & 32,8 & 4043 & 33,2 & 4324 \\
\hline Moyen & 87,2 & 1223 & 76,8 & 2984 & 79,9 & 4207 & 79,3 & 1223 & 53,5 & 2982 & 61,0 & 4205 \\
\hline Riche & 96,4 & 1353 & 89,2 & 195 & 95,5 & 1548 & 93,2 & 1348 & 78,5 & 195 & 91,3 & 1543 \\
\hline Pr. Chi2(2) & $* *$ & & *** & & *** & & $* *$ & & *** & & $*$ & \\
\hline $\begin{array}{l}\text { Fréquence d'exposition } \\
\text { aux médias }\end{array}$ & & & & & & & & & & & & \\
\hline Prat. chaque jour & 95,8 & 888 & 81,2 & 681 & 89,5 & 1569 & 91,4 & 885 & 62,4 & 681 & 78,8 & 1566 \\
\hline Au - une fois par semaine & 90,7 & 579 & 77,6 & 795 & 83,1 & 1374 & 86,2 & 578 & 53,8 & 794 & 67,4 & 1372 \\
\hline Moins d'une fois par sem. & 92,8 & 377 & 74,6 & 830 & 80,3 & 1207 & 87,0 & 377 & 50,6 & 828 & 62,0 & 1205 \\
\hline Pas du tout & 81,3 & 1008 & 63,3 & 4907 & 66,3 & 5915 & |69,2 & 1007 & 36,6 & 4906 & 42,2 & 5913 \\
\hline Pr. Chi2(3) & 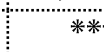 & & *** & & **⿻丷木 & & $* *$ & & $* *$ & & $*$ & \\
\hline Ethnie de la femme & & & & & & & & & & & & \\
\hline Bambara & 94,5 & 793 & 68,8 & 1980 & 76,1 & 2773 & 91,8 & 792 & 49,8 & 1975 & 61,8 & 2767 \\
\hline Malinke/Kassonké & 88,7 & 257 & 67,4 & 574 & 74,0 & 831 & 87,9 & 257 & 47,0 & 574 & 59,7 & 831 \\
\hline Peulh/foulfoulbé & 91,4 & 395 & 67,0 & 999 & 73,9 & 1394 & 83,0 & 395 & 39,4 & 999 & 51,8 & 1394 \\
\hline Sonrai & 84,4 & 295 & 69,9 & 808 & 73,8 & 1103 & 67,1 & 292 & 24,1 & 808 & 35,5 & 1100 \\
\hline Sarakole/s & 89,9 & 378 & 75,2 & 718 & 80,3 & 1096 & 83,1 & 378 & 56,0 & 718 & 65,3 & 1096 \\
\hline Sénoufo/minianka & 81,2 & 234 & 70,5 & 861 & 72,8 & 1095 & 75,6 & 234 & 57,0 & 862 & 60,9 & 1096 \\
\hline Dogon & 94,4 & 124 & 74,7 & 419 & 79,2 & 543 & 87,1 & 124 & 24,8 & 419 & 39,0 & 543 \\
\hline Maure/tamacheq/arabe & 74,8 & 155 & 30,9 & 456 & 42,1 & 611 & 58,1 & 155 & 10,3 & 456 & 22,4 & 611 \\
\hline Autres & 88,4 & 224 & 77,6 & 401 & 81,4 & 625 & 75,3 & 223 & 45,6 & 401 & 56,3 & 624 \\
\hline Pr. Chi2(8) & $* *$ & & $* *$ & & ** & & $* *$ & & $* *$ & & & \\
\hline Région & & & & & & & & & & & & \\
\hline Kayes & 86,5 & 341 & 65,1 & $117 \mid$ & 69,9 & 1512 & 80,7 & 342 & 43,6 & 1171 & 51,9 & 1513 \\
\hline Koulikoro & 90,7 & 214 & 72,9 & 1389 & 75,3 & 1603 & 86,0 & 214 & 60,7 & 1385 & 64,1 & 1599 \\
\hline Sikasso & 82,1 & 385 & 69,3 & 1535 & 71,8 & 1920 & 74,5 & 385 & 60,4 & 1535 & 63,2 & 1920 \\
\hline Ségou & 92,6 & 176 & 73,1 & 1032 & 75,9 & 1208 & 86,4 & 176 & 37,9 & 1033 & 45,0 & 1209 \\
\hline Mopti & 85,8 & 254 & 72,3 & 755 & 75,7 & 1009 & $\mid 70,1$ & 251 & 23,0 & 755 & 34,8 & 1006 \\
\hline Tombouctou & 66,4 & 140 & 64,2 & 579 & 64,7 & 719 & $\mid 52,9$ & 140 & 17,5 & 578 & 24,4 & 718 \\
\hline Gao & 86,6 & 179 & 71,4 & 454 & 75,7 & 633 & 62,6 & 179 & 24,7 & 454 & 35,4 & 633 \\
\hline
\end{tabular}


African Population Studies Vol 32, No. 3, Dec. 2018

\begin{tabular}{|c|c|c|c|c|c|c|c|c|c|c|c|c|}
\hline Kidal & 73,6 & 53 & 21,7 & 309 & 29,3 & 362 & 54,7 & 53 & 4,9 & 309 & 12,2 & 32 \\
\hline Bamako & 96,6 & 1115 & & & 96,6 & 1115 & 94,1 & 1112 & & & 94,1 & 1112 \\
\hline Ensemble & 89,3 & 2857 & 67,8 & 7224 & 73,9 & $1008 \mid$ & 81,9 & 2852 & 42,5 & 7220 & 53,7 & 10072 \\
\hline Pr. Chi2(8) & \multicolumn{2}{|c|}{ ***** } & \multicolumn{2}{|c|}{ **** } & \multicolumn{2}{|c|}{ ***** } & \multicolumn{2}{|c|}{ ***** } & \multicolumn{2}{|c|}{ ***** } & \multicolumn{2}{|c|}{$* * *$} \\
\hline
\end{tabular}

En somme, les résultats des tableaux croisés mettent en évidence d'énormes inégalités en matière de recours aux soins de santé maternelle au Mali, quelle que soit la caractéristique considérée, mais un peu moins pour l'âge de la femme. Ces inégalités sont beaucoup plus marquées pour l'accouchement assisté qu'elles ne le sont pour la consultation prénatale. Pour ces deux indicateurs, les femmes rurales sont particulièrement désavantagées par rapport à celles du milieu urbain, mais l'effet des caractéristiques varie quelque peu selon le milieu de résidence. C'est seulement en milieu rural que l'accès aux soins décline régulièrement avec l'avancement en âge. L'influence de la scolarisation diffère aussi selon le milieu. En milieu rural, c'est la minorité qui a atteint au moins le niveau secondaire qui se distingue nettement des autres, alors qu'en milieu urbain, l'effet est moins marqué et indique surtout le moindre accès de celles qui ne sont pas allées à l'école. On observe aussi une distinction entre les femmes urbaines n'ayant pas du tout accès aux médias et celles qui $y$ sont quotidiennement exposées, tandis qu'en milieu rural, le recours aux soins prénatals et obstétricaux suit le gradient du degré d'exposition des femmes aux médias. Dans les deux milieux, le recours aux soins suit le gradient du niveau de vie du ménage, mais avec un désavantage marqué des plus pauvres, surtout pour l'accouchement en milieu urbain.

\section{Analyse explicative multiniveau du recours aux soins maternels}

La régression logit dans une approche multiniveau est la méthode la plus appropriée au regard de l'objectif de démêler ce qui, dans les disparités communales d'utilisation de services de santé maternelle, relève des profils sociodémographiques de la femme ou de son ménage (facteurs individuels ou familiaux de conversion) et des facteurs collectifs de conversion (niveaux communaux d'éducation et de conditions de vie des ménages) d'une part, et d'autre part, des ressources du contexte (différences d'offre de services et spécificités de la zone de résidence). À cet effet, quatre modèles ont été estimés pour chacune des deux composantes de soins maternels (consultation prénatale et accouchement assisté) qui constituent nos variables à expliquer. Le premier est le modèle nul, incluant seulement la variable dépendante et de l'ordonnée à l'origine (ne contenant donc pas de variables explicatives). II permet de connaître la variance initiale des effets aléatoires des différences de recours aux soins entre les femmes de différentes communes et sert de référence pour le reste des modèles estimés. Le second modèle évalue les effets des variables de niveau individuel et du ménage sur les inégalités communales d'utilisation des soins. Le troisième comprend, en plus des caractéristiques individuelles et du ménage, les facteurs collectifs de conversion et les ressources du contexte. Enfin, le quatrième modèle traite des interactions entre les deux variables principales de niveau individuel et familial (éducation et conditions socioéconomique du ménage) et le milieu de résidence. Plus spécifiquement, il permet de voir si les effets de l'éducation de la femme et du niveau de vie de son ménage sont les mêmes en milieu rural et urbain.

Facteurs expliquant les inégalités communales de consultation prénatale

L'examen de la décomposition de la variance, dans le modèle nul, montre que $35 \%$ de la variabilité de la propension de suivi pendant la grossesse est imputable au fait de vivre dans des communes différentes. Ce qui révèle des inégalités importantes entre les femmes selon leur commune de résidence.

L'introduction des caractéristiques individuelles et du ménage dans la régression (modèle 2) fait baisser la variance intercommunale de I,803 à I, I89. De $35 \%$ dans le modèle nul, la part de la variabilité du recours aux soins prénatals due aux différences de communes de résidence descend à $26 \%$, soit une baisse de $52 \%$. Ce qui indique que le niveau d'instruction, l'exposition aux médias, l'ethnie, le niveau de vie du ménage, l'éducation du chef de ménage sont importants dans l'explication de la variabilité de recours aux soins selon la commune. Un peu plus de la moitié de cette variabilité est due aux caractéristiques individuelles et familiales.

Les facteurs collectifs de conversion et des ressources du contexte jouent également un rôle important dans les différences communales de recours aux consultations prénatales. L'ajout dans le modèle 3 du niveau moyen d'éducation des femmes et de celui de condition de vie des ménages, ainsi que l'offre de services de santé fait encore baisser d'un quart supplémentaire la variabilité intercommunale (baisse de la variance de I, I 89 à I,028). À l'instar des facteurs individuels et familiaux de conversion, les facteurs collectifs de conversion et les ressources du contexte jouent un rôle important dans les inégalités de recours aux soins selon la commune de résidence. 
Ce dernier modèle présente aussi les effets nets des caractéristiques individuelles et familiales, collectives et du contexte en contrôlant leurs poids respectifs. Les facteurs individuels et du ménage les plus importants à la base de ces différences communales de recours aux soins prénatals sont l'éducation de la femme et du chef de ménage, l'exposition aux médias, l'appartenance ethnique et le niveau de vie du ménage. En effet, les femmes du niveau primaire et surtout celles ayant atteint au moins le niveau secondaire présentent des propensions (rapports de chances respectifs de I,60 et 2,74 ) plus élevées de consultation prénatale que celles d'aucun niveau. Comme l'instruction de la femme, l'éducation du chef de ménage apparait aussi significative. Les femmes habitant dans les ménages dirigés par des chefs ayant atteint les niveaux primaire et secondaire ou plus sont environ deux fois plus susceptibles $(O R=I, 72)$ de recourir aux soins prénatals que celles vivant dans les ménages avec des chefs d'aucune instruction. Les femmes exposées quotidiennement aux médias sont beaucoup plus enclines à utiliser les services de santé par rapport à celles n'ayant pas du tout accès aux médias. Ces dernières ont une propension de recours aux soins prénatals de près de deux fois moins $(O R=0,62)$ élevée que chez celles quotidiennement exposées aux médias. Les femmes du groupe ethnique Maure/tamacheq/arabe ont une propension deux fois moins $(O R=0,6 \mathrm{I})$ élevée d'être suivies pendant leur grossesse par rapport à celles des autres groupes ethniques, excepté le groupe ethnique Sarakole/soninke/marka. Les femmes de ce dernier groupe présentent un avantage net $(O R=I, 30)$ par rapport à celles du groupe bambara, en termes de consultation prénatale. Excepté ces deux cas, les autres groupes ethniques ne se distinguent pas significativement des Bambaras. Les femmes vivant dans les ménages riches et, dans une moindre mesure, dans ceux de niveau de vie moyen sont plus susceptibles de recourir aux soins prénatals (rapport de chances respectifs de 3,32 et $I, 82$ ) par rapport à celles vivant dans les ménages pauvres.

II apparait, par ailleurs, que c'est dans les communes avec une proportion moyennement élevé de femmes ayant atteint au moins le niveau secondaire que le suivi prénatal est plus fréquent. En outre, on constate qu'un ratio communal élevé de services de santé accroît de près deux fois $(O R=1,46)$ le recours aux soins. $\mathrm{Ce}$ résultat exprime des différences communales considérables de dotations en infrastructures sanitaires et en personnel de santé, ce qui contribue à de fortes inégalités de suivi prénatal. Néanmoins, les inégalités de dotations en infrastructures sanitaires ainsi que dans les facteurs individuels et collectifs de conversion n'effacent pas les différences selon le lieu de résidence, à savoir le désavantage rural $(O R=I, 57)$ et particulièrement de la région de Kidal. Les femmes de Kidal sont II fois $(O R=0,09)$ moins susceptibles de recourir à la consultation prénatale par rapport à celles de toutes les autres régions, après contrôle pour toutes les autres variables.

Dans le quatrième modèle, nous examinons si les effets de l'éducation des femmes et du niveau de vie de leur ménage sur le recours aux soins prénatals varient selon leur milieu de résidence. Quelle que soit la zone de résidence, l'éducation de la femme est associée à une propension élevée d'utilisation des services de santé pendant la grossesse, comme il a été aussi observé dans les résultats descriptifs. Toutefois, l'effet de l'éducation de la femme sur l'utilisation des services de santé change selon le milieu de résidence. En effet, l'accès à l'école primaire a significativement plus d'effet sur la propension à recourir aux soins en milieu urbain qu'en milieu rural. Ainsi, les femmes urbaines du niveau primaire sont trois fois plus enclines (terme d'interaction $=3,1$ ) à recevoir des soins pendant leur grossesse que celles qui ne sont pas scolarisées. En revanche, en milieu rural, les femmes ayant atteint le niveau primaire ne se distinguent pas significativement de celles sans instruction. On observe donc une distinction entre le fait d'avoir été à l'école ou non en milieu urbain alors qu'en milieu rural, c'est la minorité de femmes ayant atteint le niveau secondaire ou plus qui se distinguent des autres (aucune éducation et niveau primaire).

Tableau 5 : Régression logistique multiniveau des effets des facteurs individuels, du ménage et contextuels sur la propension à recourir à la consultation prénatale (Logit)

\begin{tabular}{llll} 
Variables/Modalités & Vide & $\begin{array}{l}\text { Caractéristiques } \\
\text { individuelles et du et du ménage + Facteurs d'interaction } \\
\text { ménage }\end{array}$ & $\begin{array}{l}\text { Caractéristiques individuelles Modèle } \\
\text { collectifs de conversion et } \\
\text { ressources du contexte }\end{array}$ \\
\hline
\end{tabular}

PARTIE FIXE

Groupe d'âge (Jeunes)

Adolescentes

$-0.01$

$-0.01$

$-0.01$

Adultes

$-0.11$

$-0.12$

$-0.12$

http://aps.journals.ac.za 


\begin{tabular}{|c|c|c|c|c|}
\hline Variables/Modalités & Vide & $\begin{array}{l}\text { Caractéristiques } \\
\text { individuelles et du } \\
\text { ménage }\end{array}$ & $\begin{array}{l}\text { Caractéristiques individuelles } \\
\text { et du ménage }+ \text { Facteurs } \\
\text { collectifs de conversion et } \\
\text { ressources du contexte }\end{array}$ & $\begin{array}{l}\text { Modèle } \\
\text { d'interaction }\end{array}$ \\
\hline \multicolumn{5}{|l|}{ PARTIE FIXE } \\
\hline \multicolumn{5}{|l|}{ Instruction (Aucun) } \\
\hline Primaire & & $0.49^{* * *}$ & $0.47^{* * * *}$ & $0.33^{* * *}$ \\
\hline Secondaire ou plus & & $1.08^{\text {करत }}$ & $|.0|^{\pi \pi \pi}$ & $0.81^{\text {करत }}$ \\
\hline \multicolumn{5}{|l|}{ Exp. aux médias (Ch. Jr) } \\
\hline Au - une fois par sem. & & $-0.22^{*}$ & $-0.23^{*}$ & $-0.22^{*}$ \\
\hline -d'une fois par sem. & & -0.17 & -0.17 & -0.16 \\
\hline Pas du tout & & $-0.48^{\pi * \pi}$ & $-0.47^{\pi \pi *}$ & $-0.46^{\pi * \pi}$ \\
\hline \multicolumn{5}{|l|}{ Ethnie (Bambara) } \\
\hline Malinke/Kassonké & & -0.17 & -0.08 & -0.08 \\
\hline Peulh/foulfoulbé & & 0.07 & 0.11 & 0.11 \\
\hline Sonrai/djerma/haoussa & & -0.05 & 0.11 & 0.12 \\
\hline Sarakole/soninke/marka & & 0.20 & $0.26^{*}$ & $0.26^{* \pi}$ \\
\hline Sénoufo/minianka & & -0.03 & 0.01 & 0.03 \\
\hline Dogon & & 0.26 & 0.28 & 0.28 \\
\hline Maure/tamacheq/arabe & & $-0.86^{* * *}$ & $-0.49^{* *}$ & $-0.50^{* *}$ \\
\hline Autres & & 0.17 & 0.16 & 0.16 \\
\hline \multicolumn{5}{|l|}{ Niveau de vie (Pauvre) } \\
\hline Moins pauvre & & $0.67^{* * * *}$ & $0.60^{* * * *}$ & $0.58^{* * * *}$ \\
\hline Riche & & $1.45^{* * * *}$ & $1.20^{* * * *}$ & $1.26^{* * * *}$ \\
\hline \multicolumn{5}{|l|}{ Instruction CM (Aucun) } \\
\hline Primaire & & $0.56^{* * * *}$ & $0.54^{* * * *}$ & $0.53^{* \pi * *}$ \\
\hline Secondaire ou plus & & $0.60^{* * * *}$ & $0.54^{* \cdots *}$ & $0.51^{* \cdots *}$ \\
\hline \multicolumn{5}{|l|}{ Sexe du CM (Homme) } \\
\hline Femme & & 0.22 & 0.21 & 0.21 \\
\hline \multicolumn{5}{|l|}{ Instruction aggr. (Fble) } \\
\hline Moyen & & & $0.47^{\pi \pi *}$ & $0.49^{* \pi * \pi}$ \\
\hline Élevé & & & 0.37 & 0.37 \\
\hline \multicolumn{5}{|l|}{ Niv. vie Aggr. (Fble) } \\
\hline Moyen & & & 0.20 & 0.20 \\
\hline Élevé & & & 0.53 & 0.52 \\
\hline \multicolumn{5}{|l|}{ Région (Bamako) } \\
\hline Kayes & & & -0.77 & -0.74 \\
\hline Koulikoro & & & -0.33 & -0.31 \\
\hline Sikasso & & & -0.45 & -0.43 \\
\hline Ségou & & & -0.01 & 0.02 \\
\hline Mopti & & & -0.05 & -0.03 \\
\hline Tombouctou & & & -0.48 & -0.45 \\
\hline Gao & & & -0.61 & -0.59 \\
\hline Kidal & & & $-2.4 I^{* * * *}$ & $-2.38^{* * * *}$ \\
\hline \multicolumn{5}{|l|}{ Milieu (rural) } \\
\hline Urbain & & & $0.45^{* *}$ & 0.26 \\
\hline \multicolumn{5}{|c|}{ Centre santé/Hbts (Fble) } \\
\hline Moyen & & & 0.10 & 0.11 \\
\hline Élevé & & & $0.38^{* * *}$ & $0.39^{* * *}$ \\
\hline \multicolumn{5}{|l|}{ Instruction*Milieu } \\
\hline Primaire* urbain & & & & $0.79^{* * \cdots *}$ \\
\hline Sec. ou plus*urbain & & & & 0.48 \\
\hline \multicolumn{5}{|l|}{ Niveau de vie*Milieu } \\
\hline Moins riche* urbain & & & & 0.20 \\
\hline Riche* urbain & & & & 0.01 \\
\hline 4479 & & & \multicolumn{2}{|c|}{ http://aps.journals.ac.za } \\
\hline
\end{tabular}




\begin{tabular}{|c|c|c|c|c|}
\hline Variables/Modalités & Vide & $\begin{array}{l}\text { Caractéristiques } \\
\text { individuelles et du } \\
\text { ménage }\end{array}$ & $\begin{array}{l}\text { Caractéristiques individuelles } \\
\text { et du ménage }+ \text { Facteurs } \\
\text { collectifs de conversion et } \\
\text { ressources du contexte }\end{array}$ & $\begin{array}{l}\text { Modèle } \\
\text { d'interaction }\end{array}$ \\
\hline \multicolumn{5}{|l|}{ PARTIE FIXE } \\
\hline Constant & $1.25^{* * * *}$ & $1.13^{* * * *}$ & $1.05^{*}$ & $1.03^{*}$ \\
\hline \multicolumn{5}{|l|}{ PARTIE ALEATOIRE } \\
\hline Variance (SE) & 1.803 & 1.189 & 1.028 & 1.03 \\
\hline VPC (\%) & 35,40 & 26,55 & 23,81 & 23,84 \\
\hline PCV (\%) & & 51,64 & 75,39 & 75,05 \\
\hline Öbservations & 10007 & 10007 & 10007 & 10007 \\
\hline Log Likelihood & -4885.64 & -4636.69 & -4593.37 & -4587.61 \\
\hline Akaike Inf. Crit. & 9775.28 & 9317.37 & 9260.74 & 9257.23 \\
\hline Bayesian Inf. Crit. & 9789.70 & 9476.01 & 9527.55 & 9552.88 \\
\hline
\end{tabular}

Note : ${ }^{*} \mathrm{p}<0 . \mathrm{I} ;{ }^{*} \mathrm{p}<<0.05$; ${ }^{* * *} \mathrm{p}<0.0 \mathrm{I}, \mathrm{VPC}=$ Variance Partition Coefficient, $\mathrm{PCV}=$ Proportional Change in Variance

Facteurs expliquant les inégalités communales d'accouchement assisté

Les résultats obtenus avec la consultation prénatale restent quasiment valables pour l'accouchement assisté. Toutefois, comme indiqué dans les résultats descriptifs, les inégalités entre les femmes vivant dans différentes communes, en matière de recours aux soins maternels, sont plus importantes en ce qui concerne l'accouchement assisté. Un peu plus de la moitié (55\%) des inégalités du recours à l'accouchement assisté est attribuable aux différences entre les communes de résidence contre un peu plus d'un tiers $(35 \%)$ pour la consultation prénatale. La prise en compte exclusive des caractéristiques individuelles et du ménage dans la régression (modèle 2) fait baisser la variance intercommunale de 4,079 à 3,004 , soit une baisse de $36 \%$. L'introduction des facteurs collectifs de conversion et les ressources du contexte, en plus des caractéristiques individuelles et familiales, dans la régression (modèle 3 ) conduit à une baisse de $98 \%$ (baisse de la variance de 4,079 à 2,056). Ainsi, l'effet des facteurs collectifs de conversion (notamment le statut économique des femmes de la commune) et des ressources du contexte est beaucoup plus important pour l'accouchement que pour la consultation prénatale. De même, les caractéristiques individuelles et familiales jouent certes un rôle dans les inégalités communales de recours à l'accouchement assisté, mais de moindre importance que pour la consultation prénatale.

Les inégalités intercommunales sont non seulement plus marquées pour l'assistance à l'accouchement que pour le suivi prénatal, mais les facteurs de variabilités diffèrent aussi quelque peu. Après contrôle pour les autres variables (modèle complet), les femmes du groupe ethnique Maure/tamacheq/arabe, qui ont la propension de recours aux soins prénatals la plus faible, ne diffèrent pas significativement de celles appartenant aux autres groupes ethniques en termes d'accouchement assisté, excepté les Sarakole/soninke/marka. Ces dernières, tout comme pour la consultation prénatale, sont plus enclines $(O R=I, 40)$ à accoucher dans un centre de santé par rapport à celles de l'ethnie Bambara ainsi que toutes les autres.

Le sexe du chef de ménage devient significatif pour l'accouchement assisté contrairement à la consultation prénatale. Après contrôle pour toutes les variables, les femmes enceintes vivant dans les ménages dirigés par une femme ont une propension plus élevée d'accoucher avec l'assistance d'un personnel de santé $(O R=I, 5 I)$ que celles habitant dans les ménages dont les chefs sont des hommes.

Une autre particularité par rapport à la consultation prénatale réside au fait que l'effet du niveau de vie du ménage sur le recours à l'accouchement assisté varie selon le milieu de résidence, contrairement à celui de l'éducation qui ne change pas, alors que c'était le contraire pour les soins prénatals (Modèle d'interaction). Le fait de vivre dans un ménage de niveau de vie moyen n'a pas le même effet sur le recours à l'accouchement assisté dans les deux milieux. En milieu urbain, les femmes riches et de niveau de vie moyen se distinguent des plus pauvres par un meilleur accès à l'accouchement assisté, alors qu'en milieu rural, les femmes de niveau de vie moyen ne sont pas significativement différentes des pauvres. La minorité de femmes rurales vivant dans les ménages riches ont une propension de près de quatre fois (terme d'interaction $=3,9$ ) plus élevée de recourir aux soins obstétricaux par rapport aux autres (pauvre et niveau de vie moyen) du même milieu. Ce qui met en évidence le fait que des femmes ayant des caractéristiques similaires peuvent avoir des réalisations différentes en matière de recours aux soins en raison des différences d'accès aux ressources du contexte. 
Tableau 6: Régression logistique multiniveau des effets des facteurs individuels, du ménage et contextuels sur la propension à accoucher avec l'assistance d'un personnel de santé (Logit)

\begin{tabular}{lllll}
\hline Variables/Modalités & Vide & $\begin{array}{l}\text { Caractéristiques } \\
\text { individuelles et du du ménage } \\
\text { ménage }\end{array}$ & $\begin{array}{l}\text { Caractéristiques individuelles et Modèle } \\
\text { collectifs de conversion et } \\
\text { ressources du contexte }\end{array}$ \\
\hline
\end{tabular}

PARTIE FIXE

Groupe d'âge (Jeunes)

Adolescentes

$-0.08$

$-0.07$

$-0.06$

Adultes

$-0.10$

$-0.10$

$-0.10$

Instruction (Aucun)

Primaire

$0.52^{\text {क*त }}$

$0.49^{\text {*** }}$

$0.43^{\text {कबल }}$

$1.33^{* * * *}$

$1.25^{* * * *}$

$1.26^{* \pi *}$

Exp. aux médias (Ch. Jr)

Au - une fois par sem.

$-0.26^{* \pi}$

$-0.26^{\text {** }}$

$-0.25^{\text {क* }}$

-d'une fois par sem.

$-0.21^{*}$

$-0.22^{*}$

$-0.20^{*}$

Pas du tout

$-0.52^{\text {*** }}$

$-0.50^{\text {*क* }}$

$-0.47^{\text {क*त }}$

Ethnie (Bambara)

Malinke/Kassonké

0.05

0.02

0.001

Peulh/foulfoulbé

0.11

0.16

0.15

Sonrai/djerma/haoussa

$-0.44^{\text {*** }}$

0.05

0.04

Sarakole/soninke/marka

$0.23^{*}$

$0.34^{* * * \pi}$

$0.34^{\text {*** }}$

Sénoufo/minianka

0.19

0.17

0.20

$-0.41^{* *}$

0.07

0.06

Maure/tamacheq/arabe

$-0.73^{\text {**** }}$

$-0.17$

$-0.21$

Autres

$-0.08$

0.06

0.06

Niveau de vie (Pauvre)

Moins pauvre

$0.60^{* * \cdots+\cdots}$

$0.48^{* \cdots * *}$

$0.34^{* * *}$

$1.43^{\text {क*त }}$

$1.07^{* \pi \pi}$

$1.33^{\text {क*ल }}$

Instruction CM (Aucun)

Primaire

$0.30^{\text {*** }}$

$0.27^{\text {क*त }}$

$0.25^{* \pi}$

$0.69^{\text {कबत }}$

$0.59^{\text {क*त }}$

$0.57^{* * *}$

Sexe du CM (Homme)

Femme

$0.43^{\text {*** }}$

$0.41^{\pi * \pi}$

$0.40^{\text {*** }}$

Instruction aggr. (Fble)

Moyen

$0.77^{\pi * \pi}$

$0.76^{\text {क*त }}$

$0.87^{\text {*क* }}$

$0.88^{* * \pi}$

Niv. vie Aggr. (Fble)

Moyen

$0.43^{* \pi}$

$0.42^{\pi *}$

Élevé

$1.20^{\text {**** }}$

Région (Bamako)

Kayes

$-0.14$

$-0.06$

Koulikoro

0.95

1.02

0.97

1.03

Ségou

0.21

0.26

Mopti

$-0.36$

$-0.31$

Tombouctou

$-0.89$

$-0.82$

Gao

$-1.10$

$-1.02$

Kidal

$-2.67^{* \cdots *}$

$-2.62^{\text {**** }}$

Milieu (rural)

Urbain

$0.96^{* \cdots * *}$

Centre santé/Hbts (Fble)

Moyen

$0.52^{* \pi}$

$0.53^{* \pi}$

Élevé

$0.95^{\text {*क* }}$

$0.97^{\text {करत }}$

Instruction*Milieu 


\begin{tabular}{|c|c|c|c|c|}
\hline Variables/Modalités & Vide & $\begin{array}{l}\text { Caractéristiques } \\
\text { individuelles et du } \\
\text { ménage }\end{array}$ & $\begin{array}{l}\text { Caractéristiques individuelles et } \\
\text { du ménage }+ \text { Facteurs } \\
\text { collectifs de conversion et } \\
\text { ressources du contexte }\end{array}$ & $\begin{array}{l}\text { Modèle } \\
\text { d'interaction }\end{array}$ \\
\hline \multicolumn{5}{|l|}{ PARTIE FIXE } \\
\hline Primaire*urbain & & & & 0.19 \\
\hline Sec. ou plus*urbain & & & & 0.02 \\
\hline \multicolumn{5}{|l|}{ Niveau de vie*Milieu } \\
\hline Moins riche*urbain & & & & $1.03^{\pi * \pi}$ \\
\hline Riche*urbain & & & & 0.41 \\
\hline Constant & $-0.19^{*}$ & -0.17 & $-1.42^{* *}$ & $-1.45^{* *}$ \\
\hline \multicolumn{5}{|l|}{ 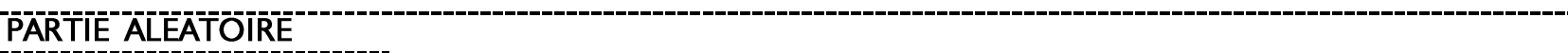 } \\
\hline Variance (SE) & 4.079 & 3.004 & 2.056 & 2.073 \\
\hline VPC (\%) & 55,35 & 47,73 & 38,46 & 38,65 \\
\hline PCV (\%) & & 35,79 & 98,39 & 96,77 \\
\hline Observations & 9996 & 9996 & 9996 & 9996 \\
\hline Log Likelihood & -4989.47 & -4706.57 & -4568.91 & -4552.56 \\
\hline Akaike Inf. Crit. & 9982.95 & 9457.14 & 9211.83 & 9187.13 \\
\hline Bayesian Inf. Crit. & 9997.37 & 9615.76 & 9478.59 & 9482.74 \\
\hline
\end{tabular}

Note : ${ }^{*} \mathrm{p}<0.1$; ${ }^{*} \mathrm{p}<0.05$; ${ }^{* * *} \mathrm{p}<0.0 \mathrm{I}, \mathrm{VPC}=$ Variance Partition Coefficient, $\mathrm{PCV}=$ Proportional Change in Variance

\section{Discussion}

Les résultats mettent en évidence des différences de recours aux soins maternels entre les communes et ceci encore plus pour le recours à l'accouchement assisté que pour le suivi prénatal. Ces différences résultent des inégalités de ressources du contexte, mais aussi des caractéristiques individuelles, familiales et des facteurs collectifs de conversion qui modulent la capacité des femmes à utiliser les services. L'effet de ces différents déterminants d'utilisation de services de santé se cumulent et contribuent respectivement à la quasi-totalité (98\%) des inégalités communales de recours à l'accouchement assisté et aux trois quarts de celles en matière de suivi prénatal. Les ressources du contexte semblent jouer un rôle plus important pour l'accouchement assisté que pour le suivi prénatal et, pour ce dernier, d'autres facteurs non considérés ici joueraient aussi un rôle (qualité de soins par exemple, autonomie de la femme, etc.).

L'effet net des facteurs individuels ou familiaux, met en évidence les inégalités locales de capacité individuelle (agencéité) à recourir aux soins selon les ressources personnelles et les caractéristiques des ménages. Les inégalités entre les communes résultent non seulement des spécificités du contexte local, mais aussi des inégalités de ressources individuelles humaines, économiques, culturelles et d'accès à l'information qui modulent la capacité des femmes à utiliser l'offre de services de santé à leurs propres fins, c'est-à-dire vivre leur grossesse dans les conditions qu'elles souhaitent. Les femmes d'un niveau d'éducation élevé, plus exposées aux médias et vivant dans les ménages d'un niveau de vie élevé sont plus susceptibles de recourir aux soins confirmant ainsi notre hypothèse $I$.

Le niveau d'éducation, un des déterminants de la demande de soins mis en évidence dans de nombreuses études (Gabrysch et al., 20 I I ; Omer et al., 2014 ; Thaddeus $S$ et Maine, etc.) peut exprimer une plus grande autonomie de la femme. Dans nombre de sociétés en Afrique, la scolarisation est le privilège des garçons. La restriction de l'accès des filles à l'école et leur abandon précoce associés à des mariages et des maternités précoces est l'expression d'une adhésion aux normes socioculturelles traditionnelles en vigueur dans ces sociétés. Avec l'éducation, la femme accède à une meilleure information et à plus d'autonomie facilitant une participation active aux processus de prise de décision relative aux questions la concernant. Ainsi elle étend ses libertés réelles de recours aux soins selon ses choix et préférences. Comme processus d'expansion de capabilités et d'autonomisation de la femme, le niveau d'éducation est d'autant plus important dans l'amélioration de la santé maternelle qu'elle lui permet de mieux planifier les grossesses et utiliser les services de santé selon sa convenance.

De même, un niveau de vie plus élevé permet aux femmes une plus grande facilité d'accès financier aux soins, sachant que les coûts de consultations et de prestations constituent un des obstacles importants de la demande de soins (Koblinsky et al., 2006 ; Peters et al., 2008 ; Perkins et al. 2009). Dans un contexte de quasi-inexistence de sécurité sociale et un système d'assurance maladie privilégiant les couches sociales les plus nanties, les libertés ou possibilités réelles de recours aux soins se trouvent être limitées chez certaines catégories socio- 
économiques, même en milieu urbain où l'offre sanitaire est supposée être suffisante et de bonne qualité. En sus, une plus grande exposition des femmes aux médias apparait importante du fait que la plupart des campagnes d'informations et de sensibilisations sont fournies par les médias, essentiellement la radio et la télévision. Indépendamment des autres facteurs, un degré élevé d'exposition aux médias contribue donc à une meilleure information sur les risques liés à la grossesse et l'accouchement d'une part, et d'autre part à une sensibilisation sur l'utilité des soins pour prévenir les complications.

Outre les caractéristiques individuelles et du ménage (facteurs individuels et familiaux de conversion), les différences communales de composition de la population féminine apparaissent tout aussi déterminantes dans les inégalités de recours aux soins. Les femmes résidant dans des communes avec une forte concentration de mères éduquées ou de ménages avec de meilleures conditions de vie sont celles qui présentent un niveau de réalisation (recours aux soins) élevé, notamment en ce qui concerne l'accouchement assisté. La concentration des femmes les plus éduquées et des ménages plus aisés reflète le niveau de développement socioéconomique d'une commune, offrant ainsi aux femmes plus de libertés ou possibilités réelles leur permettant de recourir aux soins selon leurs choix et préférences. La femme peut alors se démarquer des normes sociales et gagner en autonomie et en pouvoir de décision en matière de recours aux soins. Ces résultats sur l'effet des ressources socio-économiques collectives corroborent notre hypothèse 2 ainsi que d'autres recherches sur le sujet (Babalola et Fatusi, 2009 ; Ononokpono, 2015 ; Stephenson et al., 2006 ; Magadi et al., 2007).

Certes les facteurs de conversion, tant individuels ou familiaux que collectifs sont importants pour accroitre la capabilité de santé des femmes, mais encore faudrait-il l'existence des ressources contextuelles (DeGraff et al., 1997 ; Franckel et al, 2008 ; Entwisle et al., 1989 ; Grady et al., 1993). On constate, ici, que les inégalités de recours aux soins entre les femmes résidant dans différentes communes proviennent également des disparités communales d'ffre de services de santé et aux différences de ressources selon le milieu de résidence et la région. Ainsi, les femmes vivant dans un contexte qui leur offre plus de ressources (communes plus urbaines ou plus équipées en infrastructures sanitaires) sont plus susceptibles de recourir aux soins. Les femmes enceintes et surtout parturientes vivant dans des communes rurales sont particulièrement désavantagées en termes de recours aux soins, en ce sens que les localités urbaines concentrent l'essentiel des infrastructures sanitaires et du personnel.

La quasi-totalité des études portant sur l'utilisation des soins de santé maternelle, mettent en avant les disparités entre les milieux urbain et rural, sans véritablement chercher à faire ressortir les spécificités propres à chacun d'eux. II est vrai que le milieu rural affiche un désavantage énorme en matière de recours aux soins par rapport au milieu urbain, ceci quel que soit l'indicateur de santé maternelle considéré. Mais il n'en demeure pas moins que des particularités existent entre ces deux milieux. Comme annoncé dans notre hypothèse 3 , les effets de l'éducation de la femme (pour la consultation prénatale) et du niveau de vie du ménage (concernant l'accouchement assisté) sur le recours aux soins varient selon la zone de résidence des mères. $\mathrm{Ce}$ n'est que la minorité de femmes ayant atteint au moins le niveau secondaire qui se distinguent en milieu rural. Ce qui n'est pas le cas en milieu urbain où c'est le fait d'avoir fréquenté l'école, quel qu'en soit le niveau qui permet un meilleur recours aux soins. Comment expliquer que des femmes enceintes ayant des niveaux d'éducation similaires aient des réalisations différentes en termes de consultation prénatale, selon leur zone de résidence ? Cet écart peut provenir des différences culturelles (Merlo et al., 2005). Par exemple, le poids encore plus important des valeurs traditionnelles en milieu rural peut contribuer à limiter les libertés réelles des femmes enceintes à recourir aux soins, même si elles le désirent. Une scolarisation primaire ne serait donc pas suffisante pour permettre aux femmes rurales de se démarquer des valeurs et normes traditionnelles encore prégnantes dans ce milieu. De façon similaire, le niveau de vie du ménage affecte différemment le recours à l'accouchement assisté selon le milieu de résidence. En milieu urbain, les femmes vivant dans les ménages riches et de niveau de vie moyen se distinguent des plus pauvres par une fréquence accrue d'accouchements médicalisés, tandis qu'en milieu rural, ce ne sont que les plus riches qui se distinguent des autres

\section{Conclusion}

Dans ce travail, nous avons considéré les disparités de recours aux soins maternels en termes d'inégalités de capabilité en matière de santé maternelle, à savoir de capacité des femmes à recourir librement aux soins selon leur convenance. En examinant les inégalités communales de recours aux soins prénatals et obstétricaux par des analyses multiniveau, nous avons distingué les différences de ressources du contexte de celles dues aux moyens qu'ont les femmes pour convertir ces ressources en 
opportunités réelles de recourir aux soins selon leurs choix et préférences. Ainsi, les femmes de communes différentes n'ont pas les mêmes libertés réelles eu égard aux différences locales d'offres de services sanitaires, mais aussi de caractéristiques individuelles, du ménage et du contexte socioéconomique.

Au terme de cette analyse, il ressort que les inégalités communales d'accès aux soins maternels résultent des disparités de dotations de ressources du contexte (différences d'offres de services sanitaires et degré d'urbanisation), de facteurs collectifs de conversion (niveau moyen de scolarisation des femmes et condition de vie moyenne dans la commune) et des facteurs individuels ou familiaux de conversion (éducation de la femme, exposition aux médias, appartenance ethnique, niveau de vie du ménage et éducation du chef de ménage). Ces inégalités sont plus importantes pour l'accouchement assisté que pour le suivi prénatal. Partant du fait que l'efficacité des politiques publiques est évaluée par leur capacité à ouvrir les mêmes capabilités de santé pour tous (Ruger, 2009 ; Bonvin et Rosenstein, $20 \mathrm{II}$ ), on constate donc que les résultats des politiques et programmes en matière de santé maternelle au Mali restent mitigés. À ce jour, ils sont encore loin d'offrir les mêmes possibilités réelles c'est-à-dire la liberté pour chaque mère de recourir aux soins prénatals et obstétricaux, si elle le désire.

\section{Références bibliographiques}

Babalola, S., et Fatusi, A. (2009). Determinants of use of maternal health services in Nigeria-looking beyond individual and household factors. BMC pregnancy and childbirth, 9(I), 43.

Bonvin, J. M., et Rosenstein, E. (20I I). L'approche par les capacités comme outil d'évaluation des politiques sociales, Repenser les droits sociaux à partir de l'idée de justice. Conférence dans le cadre de la Journée Charles Gide Justice et économie : doctrines anciennes et nouvelles théories, Toulouse.

Chacko, E. (200I). Women's use of contraception in rural India : a village-level study. Health et place, 7(3), 197-208.

Chaix, B., et Chauvin, P. (2002). The contribution of multilevel models in contextual analysis in the field of social epidemiology : a review of literature. Epidemiology and Public Health/Revue d'Epidémiologie et de Santé Publique, 50, 489-99.

Congdon, P. (1995). The impact of area context on long term illness and premature mortality: an illustration of multi-level analysis. Regional Studies, 29(4), 327-344.

Courgeau, D. (2004). Du groupe à l'individu : synthèse multiniveau. Ined.
DeGraff, D. S., Bilsborrow, R. E., et Guilkey, D. K. (1997). Community-level determinants of contraceptive use in the Philippines : a structural analysis. Demography, 34(3), 385-398.

Entwisle, B., et Mason, W. M. (1985). Multilevel effects of socioeconomic development and family planning programs on children ever born. American journal of Sociology, 9I(3), 6I 6-649.

Entwisle, B., Casterline, J. B., et Sayed, H. A. (1989). Villages as contexts for contraceptive behavior in rural Egypt. American Sociological Review, I0191034.

Franckel, A., Arcens, F., et Lalou, R. (2008). Contexte villageois et recours aux soins dans la région de Fatick au Sénégal. Population, 63(3), $53 \mid-553$.

Gabrysch, S., Cousens, S., Cox, J., et Campbell, O. M. (20II). The influence of distance and level of care on delivery place in rural Zambia: a study of linked national data in a geographic information system. PLoS Med, 8(I), el 000394.

Grady, W. R., Klepinger, D. H., et Billy, J. O. (1993). The influence of community characteristics on the practice of effective contraception. Family planning perspectives, 4-I I.

Koblinsky, M., Matthews, Z., Hussein, J., Mavalankar, D., Mridha, M. K., Anwar, I., ... et Lancet Maternal Survival Series steering group. (2006). Going to scale with professional skilled care. The Lancet, 368(9544), I 377-I 386.

Magadi, M. A., Agwanda, A. O., et Obare, F. O. (2007). A comparative analysis of the use of maternal health services between teenagers and older mothers in sub-Saharan Africa: Evidence from Demographic and Health Surveys (DHS). Social science et medicine, 64(6), I 3 | I- I 325.

Manda, S. O. M. (1998). Unobserved family and community effects on infant mortality in Malawi. Genus, I43-I64.

Nganawara D. (2016). Famille et scolarisation des enfants en âge obligatoire scolaire au Cameroun : Une analyse à partir du recensement de 2005. Québec : Observatoire démographique et statistique de l'espace francophone / Université Laval, Québec, 50 p. (collection Rapport de recherche de l'ODSEF)

Nouhou, A. M., Cissé, S., Fané, D. A., Doumbia, A. G., \& Sauvain-Dugerdil, C. (20|6). Stratégies familiales et qualité de vie au Mali à travers les données du recensement. African Population Studies, 30(2).

Omer, K., Afi, N. J., Adamu, M., Malami, S. A., OyoIta, A., Cockcroft, A., et Andersson, N. (20|4). Seeking evidence to support efforts to increase use of antenatal care: a cross-sectional study in 
two states of Nigeria. BMC pregnancy and childbirth, I4(I), I.

Ononokpono, D. N. (20|5). Maternal health care in Nigeria : Do community factors moderate the effects of individual-level Education and Ethnic origin ? Etude de la Population Africaine, 29(I), I554.

Pebley, A. R., Goldman, N., et Rodriguez, G. (1996). Prenatal and delivery care and childhood immunization in Guatemala : do family and community matter? Demography, 33(2), 231247.

Perkins, M., Brazier, E., Themmen, E., Bassane, B., Diallo, D., Mutunga, A., ... et Ngobola, O. (2009). Out-of-pocket costs for facility-based maternity care in three African countries. Health policy and planning, 24(4), 289-300.

Peters, D. H., Garg, A., Bloom, G., Walker, D. G., Brieger, W. R., et Hafizur Rahman, M. (2008). Poverty and access to health care in developing countries. Annals of the New York Academy of Sciences, II36(I), |6I-I7I.

Ruger, J. P. (2009). Global health justice. Public Health Ethics, php0I9.

Stephenson, R., Baschieri, A., Clements, S., Hennink, M., et Madise, N. (2006). Contextual influences on the use of health facilities for childbirth in Africa. American journal of public health, 96(I), 84-93.

Thaddeus, S., et Maine, D. (1994). Too far to walk: maternal mortality in context. Social science et medicine, 38(8), I091-I I I0.

Vallée, J. (2009). Les disparités spatiales de santé en ville : l'exemple de Vientiane (Laos). Cybergeo: European Journal of Geography.

Von Korff, M., Koepsell, T., Curry, S., et Diehr, P. (1992). Multi-level analysis in epidemiologic research on health behaviors and outcomes. American Journal of Epidemiology, I 35(10), 1077 1082. 